\title{
LOWER BOUNDS FOR THE RADICAL OF THE GROUP ALGEBRA OF A FINITE $p$-SOLUBLE GROUP
}

\author{
by D. A. R. WALLACE \\ (Received 24th October 1967)
}

\section{Introduction and Notation}

Over a field of characteristic $p>0$ the group algebra of a finite group has a unique maximal nilpotent ideal, the Jacobson radical of the algebra. The powers of the radical form a decreasing and ultimately vanishing series of ideals and it would be of interest to determine the least vanishing power. Apart from the work of Jennings (3) on p-groups little is known in general (cf. (5)) about this particular power of the radical (cf. Remarks of Brauer in (4), p. 144, Problem 15). In this paper we give non-trivial lower bounds for the index of the least vanishing power of the radical when the group is $p$-soluble. Of the lower bounds we give we show that that lower bound, which is dependent solely on the order of the group, is the best possible such bound and that this bound is invalid if the assumption of $p$-solubility is omitted.

We consider a fixed algebraically closed field $K$ of characteristic $p>0$. The group algebra of a finite group $G$ over $K$ is denoted by $K(G)$ and $J K(G)$ is the Jacobson radical of $K(G)$. $e$ is the identity of $G$. We wish to determine the least positive integer $t(G)>0$ such that

$$
[J K(G)]^{t(G)}=\{0\} .
$$

If $p$ does not divide the order of $G$ then $J K(G)=\{0\}$ and $t(G)=1$ whereas if the order of $G$ is divisible by $p$ then $J K(G) \neq\{0\}$ and $t(G)>1$.

\section{Factor Groups}

We begin with a theorem on embeddings for which the finiteness of the group $G$ is not essential.

Theorem 2.1. Let $M$ be a normal subgroup of $G$ of order prime to $p$. Let $I$ be the ideal which is the kernel of the algebra homomorphism of $K(G)$ onto $K(G / M)$ induced by the natural homomorphism of $G$ onto $G / M$. Then there exists an ideal $A$ of $K(G)$ such that

(1) $K(G)$ is the direct sum of $I$ and $A$ and

(2) $A$ is isomorphic to $K(G / M)$.

Proof. Let

$$
G=\bigcup_{\lambda \in \Lambda} M a_{\lambda}
$$


be a coset decomposition of $M$ in $G, \Lambda$ being an indexing set. Then $I$ has a vector basis over $K$ consisting of all elements of the form $(e-x) a_{\lambda}(x \in M$, $x \neq e, \lambda \in \Lambda$ ). Let

$$
A=\left(\sum_{y \in M} y\right) K(G)
$$

Then $A$ is clearly an ideal of $K(G)$ and $A$ consists of all elements of $K(G)$ of the form

$$
\alpha_{\lambda_{1}}\left(\sum_{y \in M} y\right) a_{\lambda_{1}}+\alpha_{\lambda_{2}}\left(\sum_{y \in M} y\right) a_{\lambda_{2}}+\ldots+\alpha_{\lambda_{r}}\left(\sum_{y \in M} y\right) a_{\lambda_{r}}
$$

where $\alpha_{\lambda_{i}} \in K(i=1,2, \ldots, r)$. Let $M$ have order $m$. In the homomorphism of $K(G)$ onto $K(G / M)$ induced by the natural homomorphism of $G$ onto $G / M$ such an element as above maps onto the element

$$
\alpha_{\lambda_{1}} m\left(M a_{\lambda_{1}}\right)+\alpha_{\lambda_{2}} m\left(M a_{\lambda_{2}}\right)+\ldots+\alpha_{\lambda_{r}} m\left(M a_{\lambda_{r}}\right) .
$$

Since the cosets $M a_{\lambda_{1}}(i=1,2, \ldots, r)$ are distinct and since $m$ is prime to $p$ this element is non-zero. Thus $A \cap I=\{0\}$. To prove that $K(G)=I+A$ it is sufficient to show that if $g \in G$ then $g \in I+A$. Now $g=x_{v} a_{v}\left(x_{v} \in M, v \in \Lambda\right)$ (say) and thus

$$
\begin{aligned}
g= & -\left(e-x_{v}\right) a_{v}+a_{v} \\
= & -\left(e-x_{v}\right) a_{v}+\frac{1}{m}\left[\sum_{y \in M}(e-y) a_{v}+\left(\sum_{y \in M} y\right) a_{v}\right] \\
& \in I+A .
\end{aligned}
$$

It remains to be shown that $A$ is isomorphic to $K(G / M)$. We assert that the set $S$ given by

$$
S=\left\{\frac{1}{m}\left(\sum_{y \in M} y\right) a_{\lambda}: \lambda \in \Lambda\right\}
$$

is multiplicatively a group (of linearly independent elements of $K(G)$ ) isomorphic to $G / M$ under the mapping

$$
\frac{1}{m}\left(\sum_{y \in M} y\right) a_{\lambda} \rightarrow M a_{\lambda}(\lambda \in \Lambda) .
$$

The verification is straightforward and clearly $A=K(S)$. The isomorphism of the groups $S$ and $G / M$ then extends linearly to an isomorphism of $A$ and $K(G / M)$.

Theorem 2.2. Let $M$ be a normal subgroup of $G$ of order prime to $p$. Then $t(G) \geqq t(G / M)$.

Proof. In the notation of the previous theorem $K(G)=I \oplus A$ where $A$ is isomorphic to $K(G / M)$. Thus $A$, and hence $K(G)$, has a nilpotent ideal isomorphic to $J K(G / M)$. This establishes the inequality.

Remark. The above inequality is the best possible for if $G$ is abelian and if $P$ is a $p$-Sylow subgroup of $G$ then $J K(G)=J K(P) K(G)$ and $t(G)=t(P)$ $=t(G / M)$. 
In order to establish our next theorem we require some elementary facts concerning $p$-groups.

Lemma 2.3. Let $P$ be a p-group of order $p^{c}(c>0)$. Then

(1) $p^{c} \geqq t(P) \geqq c(p-1)+1$,

(2) $[J K(P)]^{t(P)-1}$ is spanned by $\sum_{x \in P} x$ and

(3) if $s=\sum_{x \in P} \sigma_{x} x\left(\sigma_{z} \in K, z \in P\right)$ then $s \in J K(P)$ if and only if $\sum_{x \in P} \sigma_{x}=0$.

Proof. (1) Since $\operatorname{dim} J K(P)=p^{c}-1$ we have immediately $p^{c} \geqq t(P)$. In the notation of Jennings $(3), t(P)=\Sigma \lambda d_{\lambda}(p-1)+1$ where, as $\lambda$ varies, $p^{d_{\lambda}}$ is the order of successive factor groups in a certain chain of descending normal subgroups. Thus $\Sigma d_{\lambda}=c$ and hence

$$
t(P) \geqq \Sigma d_{\lambda}(p-1)+1=c(p-1)+1 .
$$

(2) Since $P$ is a $p$-group it follows, from the definition of $t(P)$, that for all $y \in P$

$$
(e-y)[J K(P)]^{t(P)-1}=\{0\}
$$

and this implies that $[J K(P)]^{t(P)-1}$ is spanned by $\sum_{x \in P} x$.

(3) The last statement is a direct consequence of the fact that the elements $(e-y)(y \in P, y \neq e)$ form a vector basis for $J K(P)$.

Theorem 2.4. Let $P$ be a normal p-subgroup of $G$. Then

$$
t(P) t(G / P) \geqq t(G) \geqq t(P)+t(G / P)-1
$$

Proof. Let

$$
G=P a_{1} \cup P a_{2} \cup \ldots \cup P a_{n}
$$

be a coset decomposition of $P$ in $G$.

Then

$$
\begin{gathered}
K(G)=K(P) a_{1}+K(P) a_{2}+\ldots+K(P) a_{n}, \\
J K(P) K(G)=J K(P) a_{1}+J K(P) a_{2}+\ldots+J K(P) a_{n} .
\end{gathered}
$$

Now $J K(P) K(G)$ is, first, the kernel of the homomorphism of $K(G)$ onto $K(G / P)$ induced by the natural homomorphism of $G$ onto $G / P$ and is, secondly, a nilpotent ideal of $K(G)$. Thus we have the two isomorphisms,

$$
\begin{aligned}
K(G / P) & \cong K(G) / J K(P) K(G), \\
J K(G / P) & \cong J K(G) / J K(P) K(G) .
\end{aligned}
$$

Hence $[J K(G / P)]^{t(G / P)}=\{0\}$ implies that

Hence, as

$$
[J K(G)]^{t(G / P)} \subseteq J K(P) K(G)
$$

$$
[J K(P) K(G)]^{t(P)}=[J K(P)]^{t(P)} K(G)=\{0\}
$$


we have

$$
[J K(G)]^{t(G / P) t(P)}=\{0\}
$$

and therefore $t(P) t(G / P) \geqq t(G)$.

We now prove the second inequality. Since

$$
[J K(G / P)]^{\imath(G / P)-1} \neq\{0\}
$$

it follows, from the second isomorphism above, that there exists

$$
w \in[J K(G)]^{t(G / P)-1} \text { and } w \notin J K(P) K(G) .
$$

Thus if $w=s_{1} a_{1}+s_{2} a_{2}+\ldots+s_{n} a_{n}\left(s_{i} \in K(P), i=1,2, \ldots, n\right)$ then there exists $j(1 \leqq j \leqq n)$ such that $s_{j} \notin J K(P)$. Then, by Lemma 2.3.(3),

$$
s_{j}=\sum_{x \in P} \sigma_{x} x\left(\sigma_{y} \in K, y \in P\right)
$$

where $\sum_{x \in P} \sigma_{x} \neq 0$. Consider now $[J K(P)]^{t(P)-1}$ which, by Lemma 2.3. (2), is spanned by $\sum_{y \in P} y$. Then

for

$$
\left(\sum_{y \in P} y\right) w \in[J K(P)]^{t(P)-1} \quad[J K(G)]^{t(G / P)-1} \text {. But }\left(\sum_{y \in P} y\right) w \neq 0
$$

$$
\left(\sum_{y \in P} y\right) w=\left(\sum_{y \in P} y\right) s_{1} a_{1}+\left(\sum_{y \in P} y\right) s_{2} a_{2}+\ldots+\left(\sum_{y \in P} y\right) s_{n} a_{n}
$$

and in this expression of $\left(\sum_{y \in P} y\right) w$ as a sum of elements from distinct cosets of $P$ in $G$ we have, for those elements from the $j$ th coset,

Hence we have

$$
\begin{aligned}
\left(\sum_{y \in P} y\right) s_{j} a_{j} & =\left(\sum_{y \in P} y\right)\left(\sum_{x \in P} \sigma_{x} x\right) a_{j} \\
& =\left(\sum_{x \in P} \sigma_{x}\right)\left(\sum_{y \in P} y\right) a_{j} \neq 0
\end{aligned}
$$

$$
\begin{aligned}
{[J K(G)]^{t(P)+t(G / P)-2} } & =[J K(G)]^{t(P)-1} & {[J K(G)]^{t(G / P)-1} } \\
& \supseteq[J K(P)]^{t(P)-1} & {[J K(G)]^{t(G / P)-1} \neq\{0\} }
\end{aligned}
$$

and this implies that $t(G) \geqq t(P)+t(G / P)-1$.

Remark. The bounds given in the above theorem cannot be improved, for suppose that $p=2$ and that $G$ has order 4 . Let $P$ be a subgroup of order 2 . Then $t(P)=t(G / P)=2$ and so

$$
4=2.2 \geqq t(G) \geqq 2+2-1=3 .
$$

If $G$ is cyclic then $t(G)=4$ and if $G$ is the four-group then $t(G)=3$.

\section{3. $p$-Soluble Groups}

The group $G$ is said to be $p$-soluble ((2), p. 331) if there exists a normal series

$$
\{e\}=H_{0} \subset H_{1} \subset H_{2} \subset \ldots \subset H_{m}=G
$$


of subgroups $H_{i}$ of $G(i=0,1, \ldots, m)$ such that $H_{j+1} / H_{j}$ is either a $p$-group or has order prime to $p(j=0,1, \ldots, m-1)$. For such a group we have the following result.

Theorem 3.1. Let $\mathrm{G}$ be p-soluble and have a normal series $\left({ }^{*}\right)$. Then

$$
t(G) \geqq \sum_{j=0}^{m-1}\left[t\left(H_{j+1} / H_{j}\right)-1\right]+1 .
$$

Proof. We argue by induction on the number of normal subgroups appearing in a series of type (*). If $m=1, G$ is either a $p$-group or has order prime to $p$ and in either case the inequality is trivially satisfied.

Suppose $m>1$. Then $H_{1} / H_{1} \subset H_{2} / H_{1} \subset \ldots \subset H_{m} / H_{1}=G / H_{1}$ is a normal series for the $p$-soluble group $G / H_{1}$ the factors of which are either $p$-groups or have orders prime to $p$ since

$$
\left(H_{j+1} / H_{1}\right) /\left(H_{j} / H_{1}\right) \cong H_{j+1} / H_{j} .
$$

We now assume the theorem is true for $G / H_{1}$. If $H_{1}$ has order prime to $p$ then, by Theorem 2.2 ,

$$
\begin{aligned}
t(G) & \geqq t\left(G / H_{1}\right) \geqq \sum_{j=1}^{m-1}\left[t\left(H_{j+1} / H_{j}\right)-1\right]+1 \\
& =\left[t\left(H_{1} / H_{0}\right)-1\right]+\sum_{j=1}^{m-1}\left[t\left(H_{j+1} / H_{j}\right)-1\right]+1 \\
& =\sum_{j=0}^{m-1}\left[t\left(H_{j+1} / H_{j}\right)-1\right]+1 .
\end{aligned}
$$

On the other hand, if $H_{1}$ is a $p$-group, it follows, by Theorem 2.4, that

$$
\begin{aligned}
t(G) & \geqq t\left(H_{1}\right)+t\left(G / H_{1}\right)-1 \\
& \geqq t\left(H_{1} / H_{0}\right)+\left\{\sum_{j=1}^{m-1}\left[t\left(H_{j+1} / H_{j}\right)-1\right]+1\right\}-1 \\
& =t\left(H_{1} / H_{0}\right)+\sum_{j=1}^{m-1}\left[t\left(H_{j+1} / H_{j}\right)-1\right] \\
& =\sum_{j=0}^{m-1}\left[t\left(H_{j+1} / H_{j}\right)-1\right]+1 .
\end{aligned}
$$

This completes the proof.

Of all possible normal series of type $\left(^{*}\right)$ for $G$ we now consider the upper $p$-series ((2), p. 331). This is the series

$$
\{e\}=P_{0} \subseteq N_{0} \subset P_{1} \subset N_{1} \subset P_{2} \subset \ldots \subset P_{l} \subseteq N_{l}=G
$$

for which $N_{i} / P_{i}$ is the maximal normal subgroup of order prime to $p$ of $G / P_{i}$ $(i=0,1, \ldots, l)$ and $P_{j+1} / N_{j}$ is the maximal normal $p$-subgroup of $G / N_{j}$ $(j=0,1, \ldots, l-1)$. From Theorem 3.1 we have immediately the following result. 
Theorem 3.2. Let $G$ be p-soluble and have a normal series $\left(^{* *}\right)$. Then

$$
t(G) \geqq \sum_{j=0}^{t-1}\left[t\left(P_{j+1} / N_{j}\right)-1\right]+1 .
$$

Utilising this theorem we obtain a simple bound for $t(G)$ dependent only on the order of $G$.

Theorem 3.3. Let $G$ be p-soluble of order $p^{a} m,(p, m)=1$. Then

$$
t(G) \geqq a(p-1)+1 \text {. }
$$

Proof. In the above notation let $P_{j+1} / N_{j}$ have order $p^{a_{j}}(j=0,1, \ldots, l-1)$. Then $a_{1}+a_{2}+\ldots+a_{1-1}=a$ and, from Lemma 2.3. (1),

Thus, by Theorem 3.2,

$$
t\left(P_{j+1} / N_{j}\right) \geqq a_{j}(p-1)+1 \text {. }
$$

$$
t(G) \geqq \sum_{j=0}^{t-1} a_{j}(p-1)+1=a(p-1)+1 .
$$

Remark. The bound given in Theorem 3.3 is the best possible if it is merely known that $G$ is $p$-soluble of order $p^{a} m,(p, m)=1$. To see this suppose $G$ is an abelian group of order $p^{a} m,(p, m)=1$ whose $p$-Sylow subgroup $P$ is elementary abelian. Then $J K(G)=J K(P) K(G)$ and $t(G)=t(P)$. But, as $P$ is elementary abelian, it follows ((3), p. 184-185) that $t(P)=a(p-1)+1$. Hence

$$
t(G)=a(p-1)+1
$$

Theorem 3.4. Let $G$ be $p$-soluble of order $p^{a} m,(p, m)=1$ where $p$ is odd. Then $t(G)=3$ implies that $p^{a}=3$.

Proof. By Theorem 3.3,

$$
3=t(G) \geqq a(p-1)+1 .
$$

This implies that $a=1$ and $p=3$ or that $a=2$ and $p=2$ from which the theorem follows.

Remark. The result is false in the above theorem if the assumption that $p$ is odd is omitted, a counterexample being afforded by the Klein four-group.

\section{Omission of $p$-Solubility Condition}

Let $G$ be a finite group, not necessarily $p$-soluble. We shall show that the bound for $t(G)$ given in Theorem 3.3 no longer holds. In order to do this we first prove two lemmas concerning the decomposition of $K(G)$ into a direct sum of left ideals.

Lemma 4.1. Let $e_{1}, e_{2}, \ldots, e_{n}$ be orthogonal idempotents in $K(G)$ such that

(1) $e=e_{1}+e_{2}+\ldots+e_{n}$ and

(2) $K(G)=K(G) e_{1}+K(G) e_{2}+\ldots+K(G) e_{n}$

is a decomposition of $K(G)$ into a direct sum of indecomposable left ideals $K(G) e_{i}$ $(i=1,2, \ldots, n)$. As a $K(G)$-module let $K(G) e_{i}$ have $r_{i}$ irreducible modules occurring as composition factors in a composition series. Then

$$
t(G) \leqq \max \left\{r_{1}, r_{2}, \ldots, r_{n}\right\}
$$


Proof. Suppose $s_{i}$ is the least strictly positive integer such that

$$
[J K(G)]^{s_{i}} e_{i}=\{0\} \quad(i=1,2, \ldots, n) .
$$

Then the factors of the series

$$
K(G) e_{i} \supset J K(G) e_{i} \supset[J K(G)]^{2} e_{i} \supset \ldots \supset[J K(G)]^{s_{i}-1} e_{i} \supset[J K(G)]^{s_{i}} e_{i}=\{0\}
$$

are the $s_{i}$ completely reducible $K(G)$-modules

$$
K(G) e_{i} / J K(G) e_{i}, J K(G) e_{i} /[J K(G)]^{2} e_{i}, \ldots,[J K(G)]^{s_{i}-1} e_{i} /[J K(G)]^{s_{i}} e_{i} .
$$

Since the above descending chain may be refined to a composition series of $r_{i}$ factors we have $s_{i} \leqq r_{i}(i=1,2, \ldots, n)$. Let

and then

$$
s=\max \left\{s_{1}, s_{2}, \ldots, s_{n}\right\}
$$

Furthermore

$$
s \leqq \max \left\{r_{1}, r_{2}, \ldots, r_{n}\right\} \text {. }
$$

and thus

$$
[J K(G)]^{s} e_{i}=\{0\} \quad(i=1,2, \ldots, n)
$$

$$
[J K(G)]^{s}=[J K(G)]^{s} e_{1}+[J K(G)]^{s} e_{2}+\ldots+[J K(G)]^{s} e_{n}=\{0\} .
$$

Hence $t(G) \leqq s$ and this establishes the lemma.

In our next lemma and in the example that follows we employ the conventions of (1).

Lemma 4.2. Let $G$ have exactly $k$ non-isomorphic modules $F_{1}, F_{2}, \ldots, F_{k}$ and $k$ corresponding indecomposable modules $U_{1}, U_{2}, \ldots, U_{k}$. Let $C=\left(c_{\kappa \lambda}\right)$ be the Cartan matrix. Then

$$
t(G) \leqq \max _{1 \leqq \kappa \leqq k}\left\{\sum_{\lambda=1}^{k} c_{\kappa \lambda}\right\}
$$

Proof. In a composition series for $U_{\kappa}$ the irreducible modules appearing as composition factors are $F_{1}$ appearing $c_{\kappa 1}$ times, $F_{2}$ appearing $c_{k 2}$ times and so on. Thus the number of irreducible modules in a composition series for $U_{\kappa}$ is

$$
c_{\kappa 1}+c_{k 2}+\ldots+c_{k k} \quad(\kappa=1,2, \ldots, k) .
$$

The result now follows from Lemma 4.1.

Example. We consider as our group $G$ the $L F(2,5)$ for the prime $p=5$. This group is simple of order 60 and its irreducible modules have been given by Brauer and Nesbitt ((1), p. 589-590).

The Cartan matrix is here

from which, by Lemma 4.2,

$$
\left[\begin{array}{lll}
2 & 1 & 0 \\
1 & 3 & 0 \\
0 & 0 & 1
\end{array}\right]
$$

$$
t(G) \leqq \max \{3,4,1\}=4 \text {. }
$$

On the other hand the bound in Theorem 3.3 for $t(G)$ is, under the assumption of $p$-solubility,

$$
1 .(5-1)+1=5
$$

and this shows that Theorem 3.3 is false without this assumption. 


\section{REFERENCES}

(1) R. Brauer and C. NesbitT, On the modular characters of groups, Ann. of Math. (2) 42 (1941), 556-590.

(2) M. HALL, The Theory of Groups (New York, 1959).

(3) S. A. JenNINGs, The structure of the group ring of a p-group over a modular field, Trans. Amer. Math. Soc. 50 (1941), 175-185.

(4) T. L. SaATY (edit.), Lectures on Modern Mathematics, vol. I (New York, 1963).

(5) D. A. R. Wallace, Group algebras with radicals of square zero, Proc. Glasgow Math Assoc. 5 (1962), 158-159.

UNIVERSITY OF ABERDEEN 